\title{
Mujeres Con-Ciencia: una mirada a las Geociencias en Uruguay
}

Women in science: looking into the Geosciences in Uruguay

Carla Kruk

DOI: https://doi.org/10.24215/25457284e122

Limnología, Instituto de Ecologia y Ciencias Ambientales, Facultad de Ciencias y Ecología Funcional de Sistemas

Acuáticos, Centro Universitario Regional del Este,

Universidad de la República, Uruguay

ckruk@yahoo.com

(iD http://orcid.org/0000-0003-0760-1186

\section{Romina Trinchin}

Departamento de Ciencias de la Atmósfera, Facultad de

Ciencias, Universidad de la República, Uruguay

rominatrinchin@gmail.com

iD http://orcid.org/0000-0003-0511-0586

Santiago de Mello

Departamento de Ciencias de la Atmósfera, Facultad de

Ciencias, Universidad de la República, Uruguay

demello.santiago@gmail.com

iD http://orcid.org/0000-0003-2924-6111

Gabriela MVélez-Rubio

Unidad de Educación Permanente - Centro Universitario

Regional del Este, Universidad de la República, Uruguay

gabriela.velezrubio@gmail.com

(iD) http://orcid.org/0000-0002-6033-0920

Rossana Cantieri

Centro Inicial Optativo Social, Departamento de Ciencias

Sociales y Humanas, Centro Universitario Regional del

Este, Universidad de la República, Uruguay

r.cantieri@gmail.com

(iD http://orcid.org/0000-0003-2593-0717

Recepción: 08 Mayo 2019

Aprobación: 05 Abril 2020

\section{Resumen:}

Visualizar la discriminación de las mujeres en ciencia es fundamental. Aquí exploramos este proceso y su percepción en el Programa de Desarrollo de las Ciencias Básicas-Geociencias (Uruguay). Datos históricos revelaron mayor proporción de mujeres que varones entre estudiantes y egresadas/os, relación que se invirtió entre investigadoras/res especialmente en grados mayores y de dirección. Sin embargo, una encuesta mostró una baja percepción de esta inequidad. La existencia de barreras imperceptibles en la carrera universitaria y su invisibilización en el territorio universitario podrían explicar el fenómeno. Será fundamental continuar la discusión e identificar acciones para disminuir esta brecha en Geociencias.

Palabras Clave: Mujeres científicas, Uruguay, STEM.

\section{Abstract:}


Visualizing women discrimination in science is fundamental. Here we explore this process and its perception in the Development Program of Basic Sciences-Geosciences in Uruguay. Historical data revealed a higher proportion of female than male students and graduates, which decreased among professional researchers, especially in higher grades and direction roles. However, a survey showed a low perception of this inequality. The existence of imperceptible barriers and its invisibility in the space of the university might explain this phenomenon. Following this discussion will be essential to identify actions and reduce the gender gap in Geosciences.

KEYWORDS: Scientific Women, Uruguay, STEM.

\section{INTRODUCCIÓN}

Es reconocida la importancia de las mujeres en el desarrollo de la ciencia, en la innovación y en la promoción de la interdisciplina, claves del avance científico de nuestro tiempo (Nielsen et al., 2017). El pluralismo y la diversidad son dos dimensiones fundamentales para mejorar el desempeño y la creatividad, para innovar y construir una sociedad más incluyente. A pesar de ello y de las distintas acciones realizadas para estrechar la brecha científica, las mujeres aún son menos reconocidas, reciben menores salarios y alcanzan menores posiciones de dirección que los varones para el mismo trabajo o nivel de producción (Osborn, 2008; VilaConcejo et al. 2018). Estos aspectos limitan el desarrollo y los aportes de las investigadoras, y resultan en una problemática global (Shen, 2013). Las áreas vinculadas a las ciencias y tecnologías conocidas como STEM (de sus siglas en inglés Science, Technology, Engineering and Mathematics) son las más afectadas, tanto en el sector público como privado, en el ámbito educativo o empresarial (Cech y Blair-Loy, 2010).

Si bien la maternidad y el cuidado de las y los hijos son las actividades más ampliamente percibidas como limitantes de la eficiencia laboral (maternal wall o "pared maternal", Williams y Segal, 2003; Williams, 2005), los mecanismos que generan y sostienen esta problemática son variados. Estos incluyen la existencia de barreras artificiales no evidentes conocidas como glass ceiling o "techo de cristal", que limitan el avance en la carrera científica de las mujeres y otras minorías (Morrison et al., 1987; Tang, 1997; Cotter et al., 2001; Eagly y Carli, 2007; Tomassini, 2012); así como mecanismos que favorecen y/o reconocen especialmente a los varones frente a las mujeres (boys club o "club de chicos") (De Welde y Laursen, 2011). En temas de ciencia y tecnología es muy notorio el uso de estereotipos que discriminan a las mujeres respecto de su capacidad para realizar algunas tareas u ocupar algunos espacios (Vila-Concejo et al., 2018). Otras manifestaciones de inequidad de género se observan en casos de microagresiones o acosos que incluyen comentarios despectivos o acciones que son indirectas (Serio, 2016), sutiles o no, intencionales o no. Individualmente o en su conjunto, estos mecanismos generan discriminación y la necesidad de las mujeres de esforzarse más que los varones para demostrar su valor y lograr alcanzar las metas deseadas.

En Latinoamérica, también se observan estas inequidades (Yáñez, 2016). Un ejemplo es el trabajo de Rovetto y Figueroa (2017) en universidades de Argentina, que identifica obstáculos en las instituciones del "saber superior" favoreciendo la discriminación. En este contexto, Uruguay no es una excepción. Tomassini (2012) realiza un estudio comparando la construcción de las trayectorias académicas de varones y mujeres en la Universidad de la República (UDELAR). La autora plantea que existen barreras que implican recorrer caminos más complejos para las mujeres. También en Uruguay, se observa una menor representación de mujeres en el área de física y los análisis adjudican este hecho a la existencia de estereotipos, la maternidad y el cuidado de los hijos, entre otros factores (Frins et al., 2015). Recientemente, un estudio sobre el Sistema Nacional de Investigación (SNI) perteneciente a la Agencia Nacional de Investigación e Innovación (ANII) de Uruguay reveló que las mujeres tienen una menor probabilidad de ser aceptadas en este programa que los investigadores varones y, además, presentan una menor tasa de avance dentro del sistema (Bukstein y Gandelman, 2017).

En base a estudios de la Oficina de Planeamiento y Presupuesto (OPP, 2017) de la última década, las acciones en Ciencia, Tecnología e Investigación han sido puntuales e informales. A partir del año 2016, 
Uruguay participó, por un lado, en el proyecto SAGA (STEM and Gender Advancement), patrocinado por UNESCO y la Presidencia de Uruguay y, por otro lado, en el desarrollo del Programa Calidad con Equidad de Género, para reducir la brecha de género en el ámbito laboral, patrocinado por el Ministerio de Desarrollo Social, y el Instituto Nacional de las Mujeres. A partir de entonces la Universidad de la República (UDELAR) comienza a fomentar la implementación de este programa de forma voluntaria, habiéndose desarrollado al momento en diez servicios: Facultades de Ciencias Sociales, Derecho, Veterinaria, Agronomía, Ciencias Económicas y de Administración, Psicología, Humanidades y Ciencias de la Educación, Ciencias de la Información, Química y Arquitectura, Diseño y Urbanismo. Si bien en el último año se sumaron algunos servicios con perfil STEM es notoria la ausencia de servicios como las Facultades de Ingeniería o Ciencias. Para continuar conociendo el escenario actual en Uruguay y promover la aplicación de políticas públicas en Ciencia, Tecnología e Investigación que integren la equidad de género, es necesario contar con más información. Aun cuando existen esfuerzos para documentar y cuantificar la brecha de género en distintas áreas de las ciencias en Uruguay (Bielli, Buti y Viscardi, 2002; Bukstein y Gandelman, 2017), muchos de ellos en el marco del Programa Calidad con Equidad de Género, existen temáticas y espacios que no han sido abordados, actualizados o reconocidos.

El Programa de Desarrollo de las Ciencias Básicas (PEDECIBA), institución dependiente del Ministerio de Educación y Cultura y de la UDELAR, surge en Uruguay a fines de los años ochenta con el objetivo de promocionar las ciencias básicas y apoyar la formación de recursos humanos de alto nivel (Tomassini, 2012). Dentro de PEDECIBA existen distintas áreas temáticas que incluyen Biología, Física, Matemáticas, Informática, Química y Geociencias (Bielli, Buti y Viscardi, 2002). El área de Geociencias (de aquí en adelante PEDECIBA-Geociencias), creada en el año 2009, abarca el conjunto de disciplinas que estudian los procesos bio-físico-químicos relevantes al funcionamiento del sistema Tierra, en medio sólido, líquido o gaseoso y en todas sus escalas espacio-temporales. ${ }^{1}$ Comprende disciplinas que suelen agruparse dentro de la geología, la oceanografía y las ciencias de la atmósfera, aunque no se limita a ellas. Por lo tanto, es especialmente importante su contribución al conjunto de temáticas STEM de Uruguay. En general, las Geociencias presentan una menor población de mujeres en comparación con muchas otras áreas de STEM (Holmes, O’Connell y Dutt, 2015; Vila-Concejo et al., 2018).

En base a los antecedentes internacionales y nacionales de discriminación e inequidad de las mujeres en estos temas, creemos que es fundamental hacer un análisis local para aportar al mejor funcionamiento de PEDECIBA-Geociencias. En este marco, exploramos la situación de las mujeres en relación a los varones con el objetivo de analizar la problemática de discriminación e inequidad y cuáles son sus características, así como también identificar potenciales acciones para aportar a su solución. Para ello, en primer lugar, se analizaron los datos históricos de las memorias de PEDECIBA-Geociencias disponibles en su página web y, en segundo lugar, se realizó una encuesta como primera aproximación a la percepción del problema por sus integrantes: estudiantado, investigadoras e investigadores, egresadas y egresados. Así, en el marco del mes de la mujer y del Día internacional de la Mujer (8 de marzo 2018), durante las IX Jornadas de PEDECIBA Geociencias en Uruguay, se presentó el análisis de los datos históricos y se desarrolló la encuesta en tiempo real. Esto nos permitió efectuar una devolución de los resultados y su discusión, durante los dos días de desarrollo de las jornadas. En este trabajo se discuten los resultados, comparándolos con otros trabajos en la región y se describen potenciales aportes a la mejora de la situación en PEDECIBA-Geociencias.

\section{Metodología}

Se analizó la distribución de mujeres y varones en datos recabados de las memorias de PEDECIBAGeociencias disponibles en la página web. ${ }^{2}$ Esta información refiere a 170 casos incluyendo información desde el año 2009 hasta el 2018 sobre estudiantes de posgrado (maestría, doctorado), investigadoras 
e investigadores, egresadas y egresados. Para 2017, se incluye además información sobre iniciación a la investigación y pos-doctorandos.

Las y los investigadores se clasifican en asociados y activos. Estas categorías se diferencian por su grado de participación concreta en el PEDECIBA. Las y los activos poseen una participación mayor en cursos, comisiones y proyectos de investigación en el país y se clasifican en dos niveles. En el primer nivel -grado 4 y 5-, científicos y científicas tienen una línea propia de investigación, una producción de alto nivel, amplia experiencia en la disciplina y antecedentes en formación de colaboradores. En cambio, investigadoras e investigadores de segundo nivel -grado 3- presentan una producción más reciente y, bajo la dirección de aquellos de primer nivel, prestan su colaboración al desarrollo de proyectos de investigación, así como también tienen acceso a participar en la formación de tesistas.

Se evaluó el cambio temporal de la distribución de género por grado, es decir cuál es la cantidad de mujeres y de varones asignados a cada grado ( 3 a 5) por año. Finalmente se consideraron las funciones de dirección de PEDECIBA-Geociencias, incluyendo la integración de la Comisión Científica de Área (CCA) y su dirección.

En el marco de las jornadas anuales de PEDECIBA-Geociencias 2018 se realizó una encuesta en tiempo real donde participaron 55 personas ( 21 mujeres y 34 varones). La misma se desarrolló durante una actividad plenaria y consistió en acceder a través de un dispositivo móvil al sitio web live.voxvote.com en su versión básica gratuita en el cual se encontraba la encuesta incluyendo preguntas anónimas. La encuesta consistió en un total de ocho preguntas que admitían respuestas binarias del tipo sí o no, o de múltiple opción dentro de las cuales se podía seleccionar más de una opción e incluso se podía optar por no contestar la pregunta (Apéndice I). El género no fue incluido como opción en la encuesta, sino que se tomó de las y los inscriptos para la actividad de ese día. Cada pregunta era habilitada al mismo tiempo para todas la personas presentes y luego de unos segundos se inhabilitaba. Las preguntas incluyeron algunos aspectos generales de percepción de la situación laboral de las mujeres en Uruguay y en la Universidad, para luego enfocarse en la situación particular dentro de PEDECIBA-Geociencias. Se consultó, además, por posibles acciones y soluciones. Inmediatamente después de completar todo el cuestionario, se proyectaron los resultados obtenidos en forma de gráfico de barras. Allí, se pudo conocer la cantidad total de respuestas por pregunta y el porcentaje que obtuvo cada respuesta. Finalmente, se realizó un análisis y reflexión grupal en base a los resultados. Se registraron los comentarios y observaciones de las y los presentes durante la actividad. Una de las ventajas del formato elegido para realizar la encuesta es que permitió generar y devolver los resultados en forma instantánea, para luego discutir con las y los involucrados las respuestas obtenidas. La aplicación es gratuita y permite un rápido pre-procesamiento con una visualización amigable. Este método presenta como desventaja lo relativo a fallas con los dispositivos portátiles personales, demoras en la sincronización de respuesta y la necesidad de contar con acceso a internet (Wright, 2005).

\section{Resultados}

Del total de integrantes de PEDECIBA-Geociencias, considerando investigadores e investigadoras, estudiantado, egresadas y egresados se encontró una leve mayoría de mujeres (51\%, 98 casos) frente a varones (49\%, 94 casos). Al subdividir la información en estudiantes, egresados y egresadas, e investigadores e investigadoras se observaron cambios en la contribución relativa de género según la categoría involucrada (Figura 1). Del total de investigadores e investigadoras (74) las mujeres representaron la menor proporción (32\%). Sin embargo, del total de estudiantes de posgrado (96) se observó una mayoría de mujeres, llegando al 60\%. Finalmente, esta mayoría se incrementó aún más al analizar a las egresadas, alcanzando un 76\% (16). 


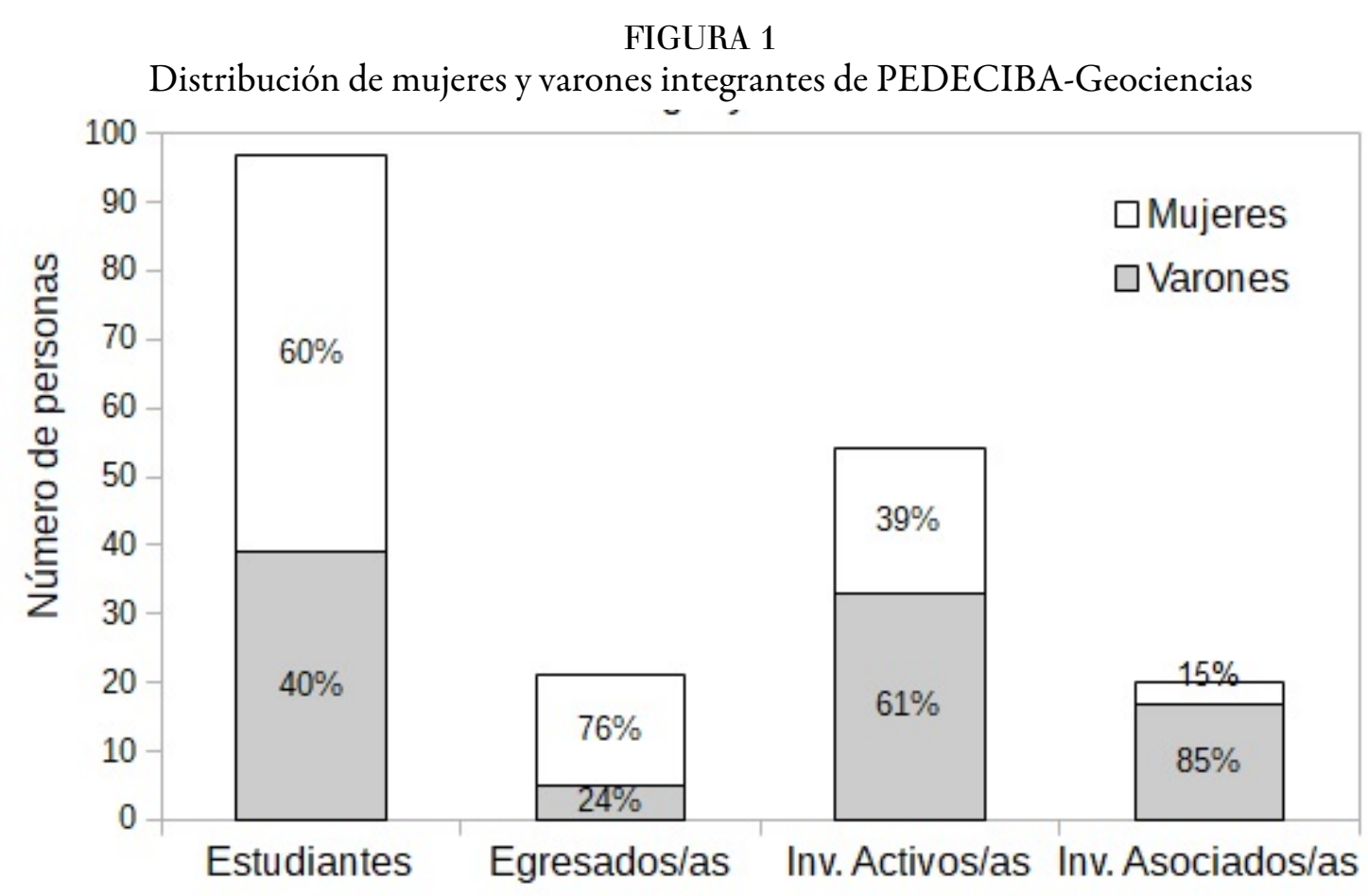

Fuente: elaboración propia en base a información de las memorias disponibles en la web desde 2009 a 2017 (http://www.pedeciba.edu.uy/geo/investigadores-geo.php).

Los y las investigadoras de PEDECIBA-Geociencias pertenecieron en su mayoría a la categoría de activos $(\mathrm{N}=54,73 \%)$ y en menor medida a asociados $(\mathrm{N}=20,27 \%)$. El porcentaje de mujeres fue mayor entre investigadores activos (39\%) que asociados (15\%) (Figura 1). Las y los investigadores asociados en general residen en el exterior y tienen una participación más limitada, mientras que las y los activos residen y/o trabajan en el país y tienen una mayor participación en la integración de comisiones, tribunales, dictado de cursos y tutoría de estudiantes.

Si consideramos investigadoras e investigadores activos y analizamos la situación actual, vemos que la proporción de mujeres varía con el grado -3,4y 5-, siendo el número de mujeres investigadoras siempre menor o igual al de varones. Esta relación en detrimento de las mujeres fue aún más importante en el grado 5 que es el grado mayor (22\%) (Figura 2A). Al analizar el desarrollo temporal de la distribución de género en relación a los grados de investigación es importante destacar que los primeros siete años de creada el área se desarrollan sin la presencia de una mujer investigadora con grado 5. Recién se visualiza el ascenso de las dos primeras investigadoras vinculadas a este grado docente, en el año 2016 (Figura 2). La dirección de PEDECIBAGeociencias, durante sus nueve años de existencia, fue siempre ejercida por varones. Asimismo, la fracción de mujeres en el Comité Coordinador Académico (CCA) fue en promedio minoritaria (40\%). 
FIGURA 2

Contribución de las mujeres a la investigación en PEDECIBA-

Geociencias según su clasificación en grados ( 3 a 5)
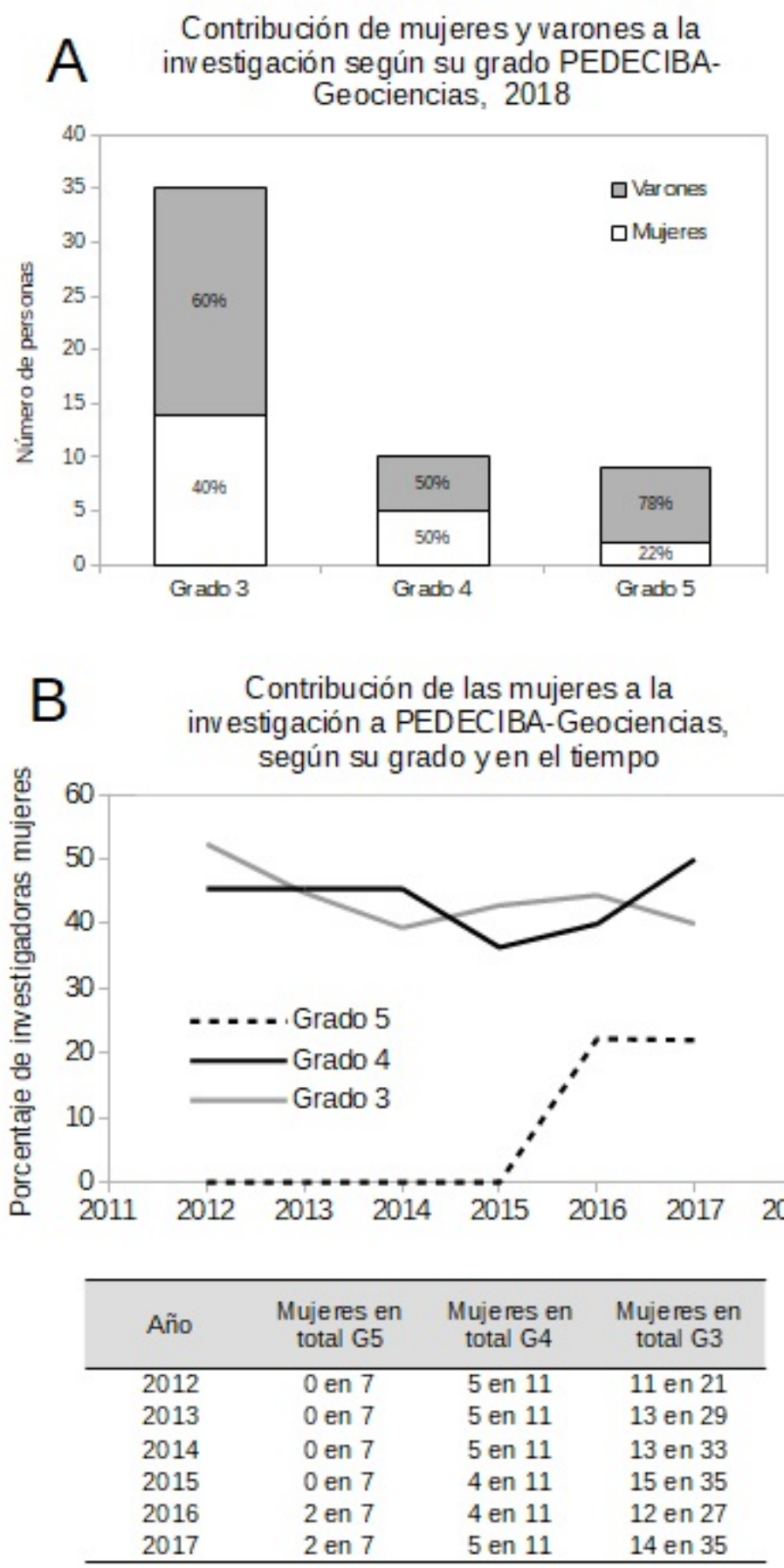

Fuente: elaboración propia en base a información de las memorias disponibles en la web desde 2012 a 2018 (http://www.pedeciba.edu.uy/geo/investigadores-geo.php).

En cuanto a la distribución de género entre estudiantes, para el año 2017 la mayoría fueron mujeres, superando en un $10 \%$ a los varones. Este patrón solo se revierte para el estudiantado de maestría que tiene una mayoría masculina (Figura 3). La mayoría femenina es especialmente notoria en el caso de los estudiantes de doctorado donde la relación mujeres/varones es de 5 a 1. Finalmente, es importante destacar que la mayor parte de las y los egresados del área son mujeres, varias de ellas actualmente están realizando su doctorado. 
En el año 2018, se otorgan las primeras becas de postdoctorado del área y resultan favorecidos una mujer y un varón.

FIGURA 3

Distribución porcentual de estudiantes de PEDECIBA-

Geociencias del año 2017 según género y nivel académico

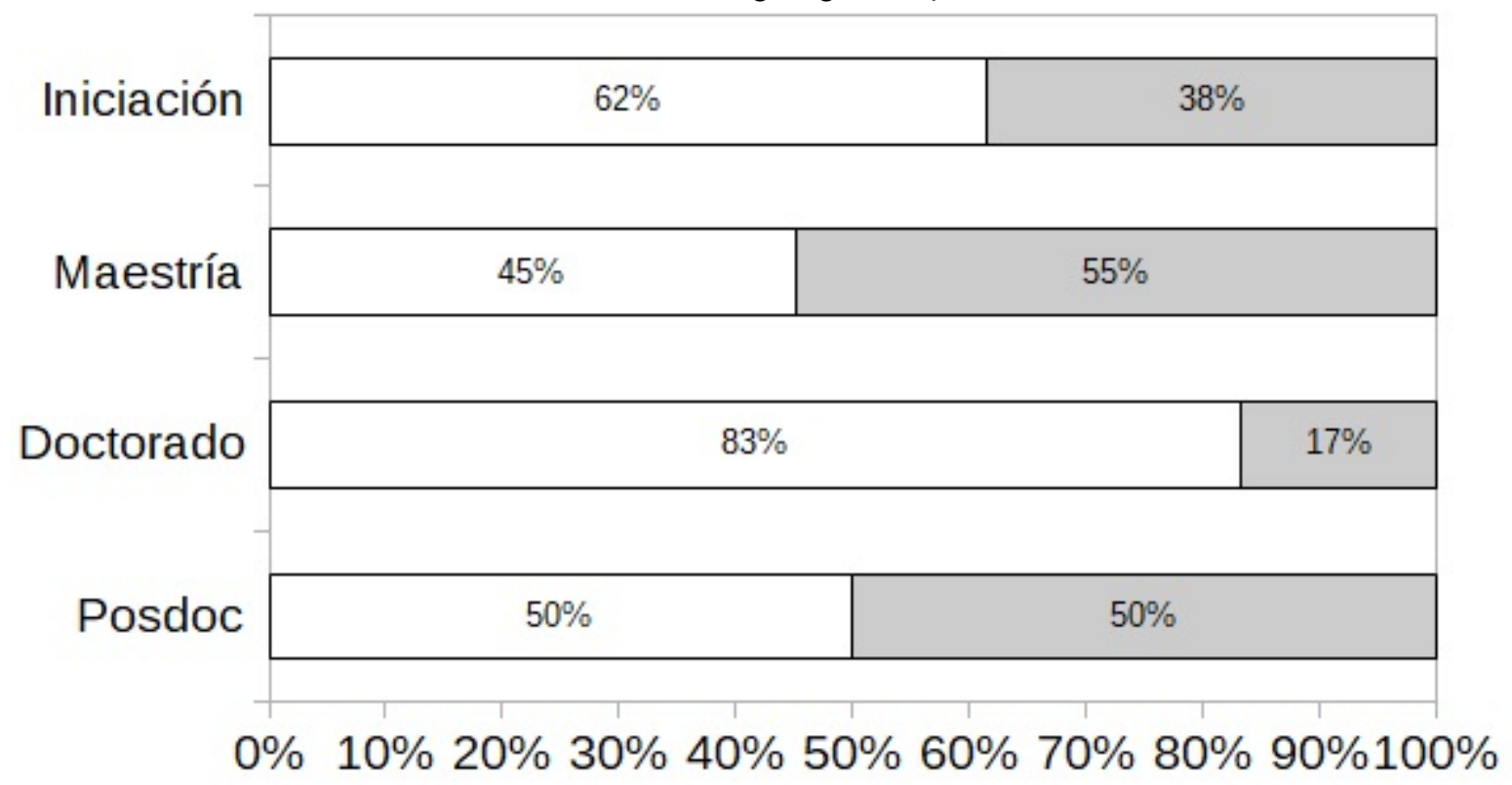

Fuente: elaboración propia en base a información de las memorias disponibles en la web. (http://www.pedeciba.edu.uy/geo/investigadores-geo.php).

\section{Percepción sobre brecha de Género}

La encuesta tuvo como objetivo conocer la percepción sobre brecha de género entre los participantes de la jornada, siendo todas y todos miembros de PEDECIBA-Geociencias. Fueron 55 los participantes de las Jornadas incluyendo estudiantes, investigadores/as, pasantes de grado de iniciación a la investigación y egresados/as, provenientes de cinco departamentos de Uruguay (Montevideo, Maldonado, Rocha, Treinta y Tres, y Salto). De estos 55 participantes, 41 (75\%) respondieron la encuesta y 14 optaron por no formar parte. Este subconjunto correspondió al 26\% del total de integrantes del área (157), con una distribución de $44 \%$ de investigadores e investigadoras, 33\% estudiantes de doctorado, 5\% de egresados, $29 \%$ de estudiantes de maestría y $86 \%$ de los pasantes de becas de iniciación. Del total de participantes, 21 fueron mujeres. Los porcentajes que se muestran a continuación se expresan sobre el total de respuestas obtenidas por pregunta.

Al inicio de la encuesta, las y los participantes reaccionaron como a un juego, generando risas y comentarios jocosos. Luego de cada consulta se proyectaban los resultados y con el correr de los mismos, las y los participantes dejaron las risas para pasar a murmurar y realizar exclamaciones de asombro ante la presentación gráfica de las respuestas a algunas preguntas.

Las preguntas una, dos y cuatro fueron relativas a la existencia de discriminación negativa hacia las mujeres en el mercado laboral de Uruguay, en la UDELAR y en PEDECIBA-Geociencias, respectivamente. A modo general, la mayoría de las y los encuestados consideró que existe algún tipo de discriminación hacia las mujeres en el mercado laboral en Uruguay $(83,8 \%, 34)$. El 58,5\% (24) opinó que la discriminación también ocurre en el contexto de la UDELAR. Finalmente, el 48,8\% (20) respondió que en PEDECIBA-Geociencias también ocurre discriminación, mientras que el 51,2\% (21) consideró que no ocurría (figura 4). Esto muestra una 
disminución del 35\% en la percepción de discriminación desde el ámbito general del Uruguay $(83,8 \%$; 34$)$ hasta el más cercano en PEDECIBA-Geociencias $(48,8 \%, 20)$ (Figura 4).

Los resultados a la pregunta “¿crees que en el área de Geociencias existe un techo de Cristal?” estuvieron en concordancia con los resultados de la percepción de discriminación de género en el área, ya que el 54,3\% (22) consideró que esta discriminación estaba relacionada con barreras no claras ("techo de cristal") dentro del área. Por otro lado, el 48,6\% (20) cree que no es así, y que la menor representación de mujeres entre los cargos de mayor nivel podría deberse a otras causas no relacionadas con discriminación de género.

FIGURA 4

Encuesta sobre percepción de discriminación de la mujer. PEDECIBA-Geociencias. Uruguay. 2018

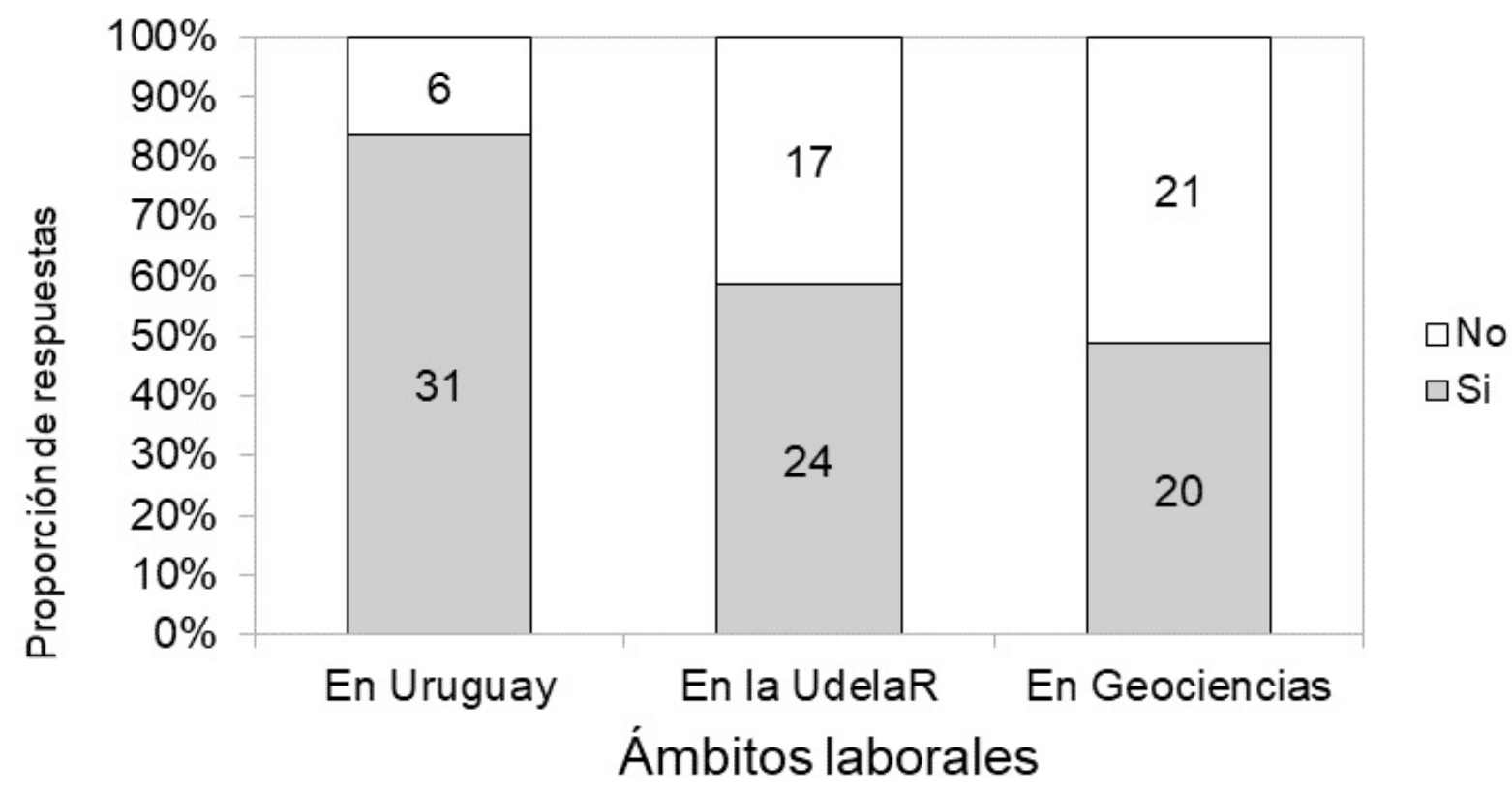

Fuente: gráfico de elaboración propia en base a la encuesta realizada en las IX Jornadas de PEDECIBA Geociencias en Rocha, Uruguay marzo 2018.

Luego se preguntó: “¿En qué sentido crees que las mujeres son discriminadas en el trabajo?”, incluyendo las opciones: menor salario, peores puestos de trabajo, privación de la promoción en el trabajo, falta de respeto por parte de los compañeros, cuestiones legales, mayor dificultad para encontrar trabajo, o falta de preparación para trabajos cualificados. El principal aspecto identificado como forma de discriminación hacia las mujeres en el trabajo fue la privación de la promoción $(27 \%, 24)$, seguido de la ubicación en peores puestos de trabajo $(21 \%, 19)$ y el menor salario $(18 \%, 16)$ (Figura 5$)$. La "falta de respeto hacia las compañeras" (15\%, 13) y la dificultad para encontrar trabajo $(15 \%, 13)$ también fueron procesos relevantes de acuerdo a la percepción de los integrantes de PEDECIBA-Geociencias. La expresión "falta de respeto" fue utilizada por su valor coloquial, nuestra intención fue reflejar en ella aspectos de microagresiones o violencia no física hacia las mujeres. 
FIGURA 5

Respuestas encuesta PEDECIBA-Geociencias sobre principales formas de discriminación de las mujeres

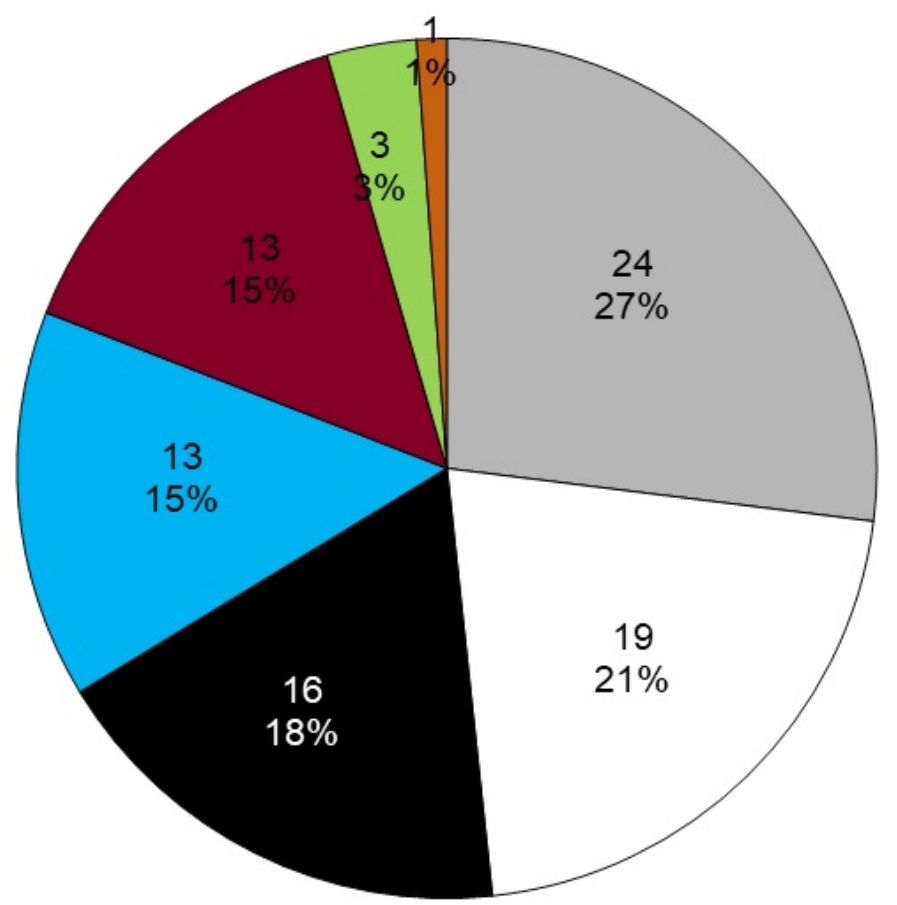

\author{
$\square$ 1. Privación de la \\ promoción \\ $\square 2$. Peores puestos de \\ trabajo \\ घ3. Menor salario \\ $\square$ 4. Mayor dificultad para \\ encontrar trabajo \\ -5. Falta de respeto por \\ compañeras \\ $\square 6 . C u$ estiones legales
}

$\square 7$. Falta de preparación para trabajos cualificados

Fuente: elaboración propia en base a la encuesta realizada en las IX Jornadas de PEDECIBA

Geociencias Uruguay en Rocha, Uruguay marzo 2018. Se incluye para cada categoría

el número total de respuestas y su porcentaje, para un total de 41 encuestados/as.

$\mathrm{Al}$ preguntarles " ¿Crees que es necesario tomar acciones concretas en cuestiones de género dentro del área?” -pregunta 5-, el 69\% (27 respuestas) de las y los encuestadas estuvo de acuerdo con la necesidad de tomar acciones concretas en cuestiones de género dentro del área, mientras que el 31\% (12) opinó que no era necesario. Esto no coincide con la idea reflejada por el total de las y los encuestados en relación a las preguntas en el ámbito laboral general donde los porcentajes de reconocimiento de la existencia de discriminación laboral y de necesidades de acciones era mayor al $80 \%$.

Finalmente, -pregunta 6- se interrogó: “¿Cuál crees que es la primera medida a tomar para mejorar la situación de la mujer dentro del área?”, con las siguientes opciones de respuestas: que exista equidad en las comisiones, que se considere la maternidad o que los proyectos incluyan un cupo mínimo de mujeres participantes. Del total de 41 encuestados, solo 34 (83\%) respondieron esta pregunta, probablemente asociado a que más del 30\% no consideró necesario tomar medidas en el ámbito propio de PEDECIBAGeociencias. Dentro de este $83 \%$ que estuvo de acuerdo con la necesidad de acciones, la existencia de equidad en las comisiones y las consideraciones respecto a la maternidad fueron marcadas por el $70,3 \%$ de las personas encuestadas (24). 
FIGURA 6

Distribución de respuestas sobre la primera medida a ser tomada para revertir inequidades de género entre miembros del PEDECIBA-Geociencias

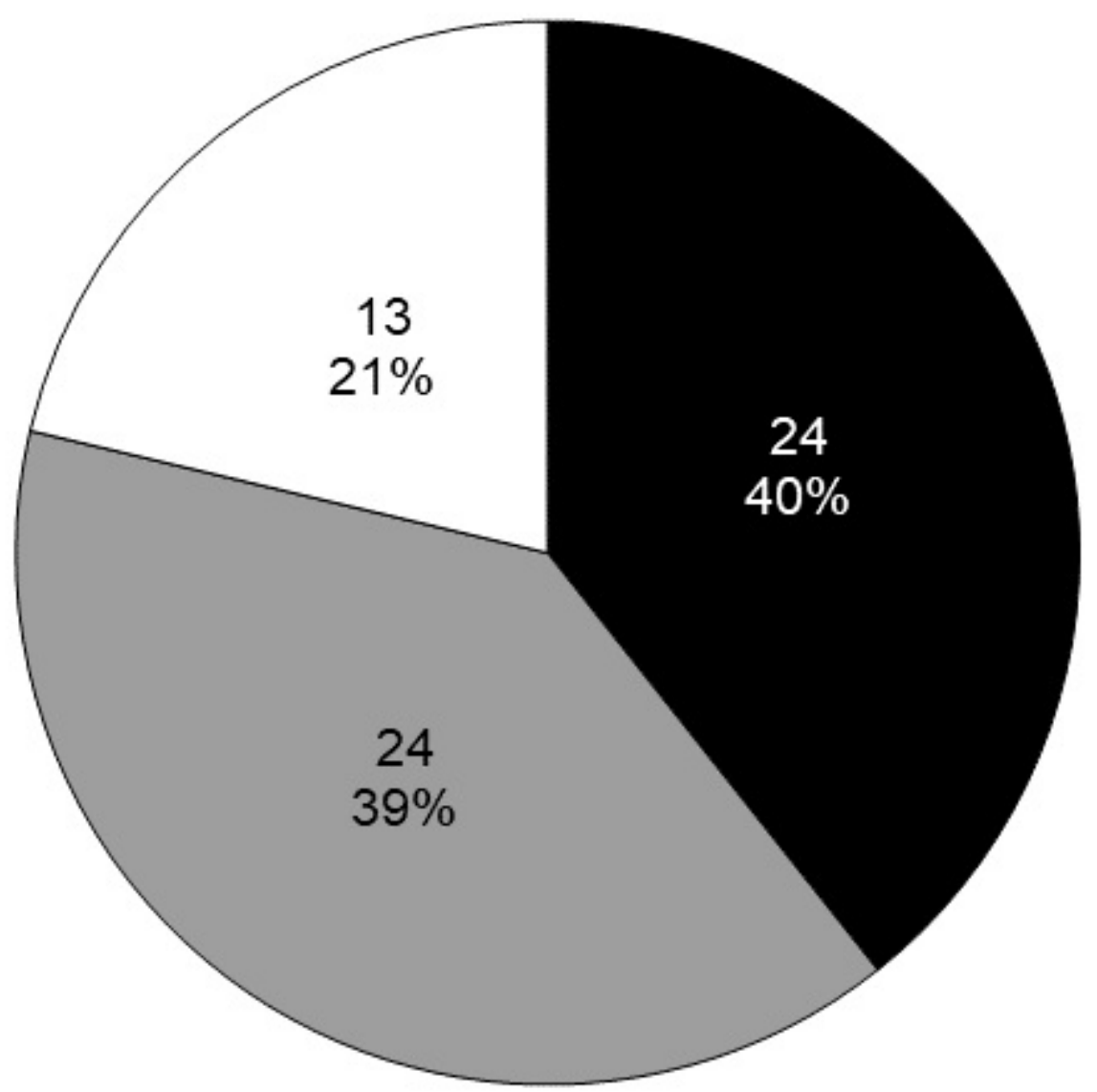

-1. Equidad en las comisiones

$\square$ 1. Considerar maternidad $\square 2$. Proyectos con igualdad de género

Fuente: elaboración propia en base a la encuesta realizada en las IX Jornadas de PEDECIBA Geociencias en Rocha, Uruguay marzo 2018. Se incluye para cada categoría el número total de respuestas y su porcentaje, para un total de 41 encuestados/as.

\section{Discusión}

En este trabajo exploratorio se documentaron y cuantificaron algunos de los componentes de la brecha de género (y otras posibles inequidades) en el área de Geociencias de PEDECIBA. Para ello recabamos datos históricos y realizamos una encuesta de percepción acerca de la problemática de género entre las y los 
integrantes del área. En el análisis histórico, la distribución de las mujeres fue equitativa considerando todos y todas las integrantes del área. Sin embargo, al desglosar por roles se identificó una distribución sesgada (Figura 1). La proporción de mujeres fue mayor entre estudiantes y egresados/as, y disminuyó notoriamente entre investigadores/as, especialmente entre las y los de mayor grado, no encontrándose mujeres en los roles de dirección (Figura 2).

Por otra parte, las y los integrantes del área, que participaron de la encuesta, percibieron la existencia de limitaciones al desarrollo profesional y laboral de las mujeres. Pero esta percepción, si bien resultó clara a nivel del país, se fue desdibujando cuando el foco se hizo en el propio grupo de científicos y científicas (Figura 4). Estos resultados, en conjunto, indicarían que existe la problemática de inequidad de género, con un claro patrón de limitación a nivel de las investigadoras, pero no una clara percepción del problema. Estos hallazgos van en el mismo sentido que otras investigaciones en la región (Tomassini, 2012; Rovetto y Figueroa, 2017) y en el mundo (Vila-Concejo, et al. 2018). Por lo tanto, resulta fundamental visualizar y difundir estos resultados, para discutirlos y evaluar los potenciales procesos estructuradores.

Los datos de baja representatividad en posiciones académicas mayores y cargos de dirección observados en PEDECIBA-Geociencias (Figura 2A y B) son congruentes con la situación internacional de las mujeres científicas. A nivel mundial, se reconoce la sub-representación de las mujeres en las direcciones universitarias, en los equipos de investigación y en los doctorados, generando una brecha en su representación en puestos de decisión o en las posiciones más altas de la academia (Osborn, 2008; Shen, 2013).

A diferencia de lo que ocurre con las investigadoras, el número de mujeres es mayor que el de varones entre estudiantes (Figura 1). Esto concuerda con los datos recabados para el desarrollo de la Estrategia Nacional para la igualdad de género 2030 (Consejo Nacional de Género) que indican que el nivel educativo terciario es alcanzado en el 22,3\% de las mujeres versus el 16,1\% de los varones, con un total de $68 \%$ de mujeres entre las y los egresados de la UDELAR en el 2013, lo cual también se condice con nuestros resultados que muestran un $76 \%$ de mujeres egresadas (Figura 1). Esto podría ser un resultado alentador, si pensamos que esta composición relativa podría luego trasladarse como "masa crítica", a la categoría de investigador permitiendo disminuir la brecha de género (Osborn, 1994). En concordancia, estudios previos señalan que las condiciones de inserción actual de las mujeres han mejorado y que el recambio generacional podría equiparar las desigualdades (OPP, 2017).

Sin embargo, un aumento modesto en el número de mujeres, sin un cambio en la estructura y el funcionamiento de la institución científica, crea lo que se denomina una "paradoja en la hipótesis de la masa crítica", en la que un aumento de las egresadas no disminuye la brecha de género (Etzkowitz et al., 1994). En este sentido, es posible comparar nuestros resultados con los obtenidos por Bielli, Buti y Viscardi (2002) para el periodo 1995-1999 para el total de PEDECIBA y teniendo en cuenta que Geociencias no existía. En ese período, la proporción de mujeres investigadoras activas era del 38,5\% siendo muy similar al 39\% relevado en este trabajo (Figura 1). De manera similar, la proporción de mujeres en el nivel 1 (cargos 4 y 5) era 27,5\% siendo 22\% sólo considerando los grados 5 en nuestros resultados (Figura 2). Una subrepresentación en comisiones y dirección también fue constatada en aquel momento. Si bien en nuestros resultados se observa un leve aumento de la proporción de mujeres en la formación de posgrados (60\% respecto del 53,4\% en 1995-1999) (Figura 3), para el resto de las categorías la situación no parece ser muy distinta a la relevada hace 20 años.

Las causas de la segregación vertical se han asociado a las tensiones entre la vida personal y laboral en la etapa temprana de la carrera y se expresan en el aumento de la longitud de la trayectoria, abandono del campo de investigación o disminución del número de hijos, observado en distintos países de Latinoamérica (Yáñez, 2016). De forma similar, en Uruguay y en base a datos del SNI, las mujeres requieren hasta cuatro años más de trayectoria para alcanzar el mismo nivel, especialmente en ingeniería y tecnología, áreas donde disminuye aún más la proporción de mujeres a mayor nivel jerárquico (Bernheim, 2015). Tomassini (2012) observa para la mujer en la universidad, conflictos entre el cuidado y la dedicación profesional. Además, la autora identifica 
la presencia de estereotipos en la división del trabajo, donde las mujeres participan con mayor intensidad en las tareas de enseñanza y extensión, actividades que obtienen menores puntajes que la investigación en la evaluación global.

Otros trabajos han identificado procesos presentes en el sistema de investigadores, muchas veces independientemente de los aspectos particulares de las mujeres. Este tipo de mecanismos, como por ejemplo el "techo de cristal" o glass ceiling que impone limitaciones no explícitas al avance profesional de las mujeres, predice que al entrar en la carrera científica las nuevas investigadoras se verán tan limitadas como las actuales (Cotter, et al, 2001). En las últimas décadas y en todo el mundo, la incorporación de las mujeres a la universidad ha aumentado significativamente. Sin embargo, la proporción de mujeres en el total de investigadores se invierte al revisar grupos académicos de egresados/as activos/as, generando lo que se denomina "efecto tijera" (Van Vlooten, 2005). Este efecto refiere a la trayectoria académica de las mujeres, en la que la presencia mayoritaria de ellas durante su formación universitaria se va revirtiendo según ascienden las responsabilidades y cargos, mientras aumenta el número de varones. En PEDECIBA-Geociencias, el 60\% del estudiantado son mujeres, $39 \%$ investigadoras ( $22 \%$ en grado 5 y ninguna en dirección) y $76 \%$ son egresadas del programa que no han iniciado su carrera como investigadoras aún (Figura 1). En España, el número de mujeres estudiantes universitarias llega al 56\% del total, pasando a ser un $45 \%$ del profesorado, un $28 \%$ de las cátedras y un $8 \%$ de las rectoras de universidades públicas y privadas (Unidad de Mujeres y Ciencia, 2018). En nuestra región se observan patrones similares. Por ejemplo, los miembros de la Academia de Ciencias de Brasil son en su amplia mayoría varones, a pesar de que varones y mujeres tienen en promedio el mismo número de publicaciones por año y que las mujeres conforman más recursos humanos que los varones (Ferrari et al., 2018).

En cuanto al análisis de la percepción de los integrantes del área respecto de la brecha de género, se encontró un reconocimiento del problema a nivel del país y de la Universidad, pero este no fue tan claro dentro de PEDECIBA-Geociencias. Esto se deduce de la disminución del 35\% en la percepción de discriminación desde el ámbito general del Uruguay (83,8\% -31-) hasta el más cercano en PEDECIBA-Geociencias (48,8\% -20-) (Figura 4). Los principales aspectos identificados como forma de discriminación hacia las mujeres en el trabajo fueron la privación de la promoción, la ubicación en peores puestos de trabajo y los menores salarios (Figura 5). La falta de respeto hacia las compañeras (como indicador de violencia no física) y la dificultad para encontrar trabajo también fueron procesos identificados como relevantes. Estas preguntas refirieron a la situación de las mujeres en general y no específicamente en PEDECIBA-Geociencias.

Preocupa que no se identifique la problemática dentro de PEDECIBA-Geociencias, elemento fundamental para la generación de acciones y soluciones a la misma (Fraser, 1997). En relación a este aspecto, traemos la reflexión de Martinis (2012) en un trabajo sobre violencias en el sistema educativo. En el mismo señala como central el acercarnos a diversas formas de "construcción del otro", que entra en escena cuando, como actores, miramos a los otros que no forman parte de nuestro grupo. Desde allí deja entrever aquello que "la violencia está en los otros", esa "otredad" es la que produce la violencia y la que se ve afectada por ella. En el caso que nos compete, es ese espacio que está más allá del nuestro, el que produce desigualdad e inequidad, que constituye formas solapadas de violencias e inequidades observadas fuera del ámbito de trabajo diario. Sin embargo, se invisibilizan las problemáticas en el territorio propio.

Coincidimos con Martinis (2012) en que la construcción de teoría no debería entenderse como un ejercicio abstracto y neutro, sino "como un esfuerzo de construir herramientas que permitan significar los fenómenos sociales e intervenir en ellos" (Martinis, 2012, p. 7). Desde este sentido, recupera aportes críticos trabajados por Bourdieu, Passeron, Melandres y Subirats (1977) sobre nociones de "violencia simbólica y arbitrariedad cultural", haciendo hincapié y llamando la atención sobre múltiples formas de destrato que van más allá de agresiones físicas y que tienen tantas o peores consecuencias que estas. Asimismo, incorpora en esta reflexión los conceptos de violencia institucional e incivilidades que trabaja ampliamente Nilia Viscardi (1998, 1999, 2003, 2008). Esta autora alerta sobre las dificultades para detectar e identificar qué es la 
violencia. Es así que detalla dos tipologías: Por un lado, la más reconocida: la violencia física que se manifiesta en golpes, heridas, etc. Por el otro, la violencia como un conjunto de incivilidades, de agresiones cotidianas al derecho de cada quien a ver su persona respetada: palabras hirientes, interpelaciones, humillaciones, etcétera. Estas incivilidades están presentes en diferentes ámbitos de convivencia y son muchas veces naturalizadas e incluso negadas en su carácter de actos violentos. Se trata entonces de una violencia institucional o institucionalizada.

Otra mirada complementaria agrega un análisis pertinente de esta forma de observación/reacción desde quienes se involucran directamente en la percepción de estas desigualdades (pues son parte de las áreas trabajadas en el presente estudio). Esta es la de Almudena Hernando quien plantea que la principal trampa del orden social para legitimar las desigualdades consiste en enseñar a todos los miembros a mirar el mundo a través de la mirada particular de quienes tienen el poder. Semejante punto de vista "se inviste de universalidad y a través de las instituciones de enseñanza, información y publicidad (...) se convierte en una verdad que toda la sociedad reproduce, aunque no se ajuste a la experiencia de quienes no ocupan esa posición de poder" (Hernando, 2015, p. 16).

Estas reflexiones nos ayudan a comprender algunas de las situaciones de invisibilidad/negación de parte de este colectivo académico que experimenta una o varias de las desigualdades antes detalladas. Esta trampa deja ver cómo opera el discurso desde el poder-saber dominante, discurso de verdad, naturalizado y asumido como única realidad. Se da el caso de que muchas de las situaciones que se presentaron como parte de los resultados de las encuestas y otras muestras con las cuales se trabajó, tienen una experiencia de vida que contradice esa verdad (que por otro lado siguen reproduciendo), en donde todas y todos tienen las mismas oportunidades y accesos. Si no se llega a ocupar algunas de las posiciones de poder/jerarquía o éxito, como se mencionaba, es porque no tienen las capacidades o cualidades, profundizando el efecto perverso de atribuir desvalorizaciones a quienes no pertenecen a ese espacio dominante o de poder (Hernando, 2015, pp. 16-17).

Hernando plantea que es necesaria una reflexión colectiva, que permita detectar o visibilizar esas formas de reproducción, dispositivos a través de los cuales el orden dominante se sigue desarrollando en la actualidad. Para ello, la autora sugiere "profundizar en las estrategias tanto discursivas, como prácticas, sexuales o científicas" que puedan ayudar a deconstruir, desandar, revertir esos dispositivos. Así también habla de dispositivo y recoge lo reflexionado por Fátima Arranz sobre Foucault, en donde refiere "una red de relaciones entre elementos heterogéneos (discursos, instituciones, arquitecturas, reglamentos, enunciados científicos, etc.), en la que el propio dispositivo establece la naturaleza del nexo de la red; nexo que liga a todos los elementos heterogéneos" y los orienta en una dirección (Hernando, 2015, pp. 20-21). Esta red de relaciones (dispositivos) no son conscientes para quienes los llevan a la práctica, son esa visión del mundo y de la realidad que siempre aparenta ser objetiva, neutral y natural, pero que vemos que no lo es. De allí la importancia de desvelar y desnaturalizarlas. $\mathrm{Al}$ momento de preguntar sobre propuestas para paliar efectos de diferencias por género dentro de PEDECIBA-Geociencias la gran mayoría, es decir, más del 60\%, estuvo de acuerdo en aplicar medidas para disminuir la brecha de género. Esto indica cierta disrupción entre el reconocimiento explícito del problema y la disponibilidad a tomar acciones al respecto.

En este marco, Rovetto y Figueroa (2017) analizan cuáles podrían ser los mitos que favorecen o permiten la existencia de inequidad de género y discriminación hacia las mujeres en el territorio universitario. En particular, los mitos que expresan esta otredad son "quienes accedemos a la educación superior no ejercemos ni padecemos violencia" y "la universidad es un lugar de avanzada en la elaboración de ideas y democrático en sus prácticas". Ambos mitos planten una comunidad idealizada y abstraída de las problemáticas globales, existen dentro de la comunidad universitaria y son causa y barrera a la hora de visibilizar las problemáticas de género en el propio ámbito. Una señal de esto se desprende del análisis de la encuesta de percepción donde semejantes problemáticas son visualizadas a nivel país, pero no en torno a una comunidad académica más reducida, como sería el caso del programa PEDECIBA-Geociencias. 
Será necesario profundizar en los análisis de estos aspectos que, entre otras cosas, permitirán preparar actividades de reflexión y dinámicas para la selección y puesta en práctica efectiva de medidas tendientes a la disminución de la brecha/desigualdad de género. Nuevos abordajes serán dirigidos a la evaluación de la heterogeneidad de la percepción entre varones, mujeres y otras identidades, por edad y por rol en el área, ya que no se tomaron en cuenta en la encuesta realizada.

De nuestros resultados preliminares, la toma de consideraciones respecto a la maternidad surge como uno de los aspectos más relevantes a tomar en cuenta para disminuir la brecha. En el ámbito de las ciencias, así como en general en Uruguay, las principales políticas implementadas en cuanto a inequidad de género refieren a la maternidad (Oficina de Planeamiento y Presupuesto, 2017). En el SNI, se contempla un año sin producción para las mujeres en período maternal (Etchebehere, 2019). En PEDECIBA central existe una normativa que permite por razones fundadas, como por ejemplo la maternidad, solicitar una prórroga en el plazo de evaluación. En PEDECIBA-Geociencias se ha implementado una licencia especial por maternidad para estudiantes de posgrado. Estas opciones no son de amplio conocimiento por las integrantes del PEDECIBA y, por lo tanto, su difusión es relevante.

Sin embargo, la maternidad no es el único factor que incide en la segregación a las mujeres. El sistema científico, sus instituciones, sus relaciones internas, los estereotipos y otros procesos sustentan el funcionamiento de género hegemónico tradicional. En nuestro caso, surge la inequidad de género en las comisiones como problema percibido, y en cargos altos y de dirección como problemáticas identificadas (Figura 6). En este sentido, sería deseable buscar mecanismos que favorezcan la participación de las mujeres en estos ámbitos, como por ejemplo implementando cupos mínimos en cargos de dirección y comisiones. Estas medidas podrían estar inspiradas y en sintonía con la legislación vigente relativa a la temática como es la "Ley de cuotas" (ley No 18.476, de 2009) que promueve y obliga la intervención de las mujeres en política, propiciando su acceso a cargos de poder y su participación en espacios formales de decisión, en iguales condiciones que los varones. En el SNI, recientemente, se han incorporado más mujeres en las comisiones de evaluación lo que ha resultado en que más mujeres subieran de categoría (Etchebehere, 2019). Dentro de posibles alternativas a discutir, se podrían incluir el financiamiento de proyectos específicos desarrollados por mujeres para promover su desarrollo en áreas especialmente deprimidas (o sin mujeres) y la inclusión de espacios extrafamiliares dedicados al cuidado, apoyando la participación de las integrantes con hijas e hijos en las actividades del área.

Actualmente, en Uruguay está vigente la Estrategia Nacional para la Igualdad de Género 2030 (Consejo Nacional de Género, 2020). Una de sus aspiraciones involucra al Sistema Nacional de Educación Pública (punto $\mathrm{V}$ de la estrategia) promoviendo varios aspectos que nos conciernen: el incremento del acceso de las mujeres a áreas STEM, la visibilización del conocimiento generado por las mujeres, el desarrollo de alianzas entre las instituciones y grupos para estimular trayectorias de mujeres, y la universalización de la educación integral en sexualidad y en violencia basada en género en el sistema educativo. En este marco, la aplicación del actual programa de calidad con equidad de género llevado adelante por el Ministerio de Desarrollo Social en conjunto con universidades y organismos públicos podría ser una alternativa válida a llevar a cabo por PEDECIBA-Geociencias. Esta guía permitiría aplicar de manera estandarizadas los resultados y acciones derivadas de este trabajo y de la Estrategia Nacional, permitiendo su comparación con similares procesos en otros servicios y grupos académicos. Sin embargo, surgen algunas limitaciones a la implementación del modelo. En primer lugar, dentro del colectivo analizado, no hay una identificación del problema, por lo tanto, no se percibe la necesidad de buscar un mecanismo que permita llegar a equidad de género en el programa. Tal vez, esto implique en primera instancia generar etapas previas de visibilización y sensibilización del problema. Asimismo, la limitación económica y de recursos humanos dificulta llevar adelante la implementación y mantener un seguimiento. 


\section{REFLEXiones FINALES Y PERSPECTIVAS}

La incorporación equitativa de las mujeres y su potencial intelectual es fundamental para el avance de la ciencia (Guevara, Medel y García, 2012; Cárdenas Tapia, 2015). En la actualidad, uno de los principales intereses de PEDECIBA-Geociencias es avanzar en la investigación interdisciplinaria (PEDECIBA Geociencias, 2018), una forma preponderante de investigación científica en nuestro tiempo (Nielsen et al., 2017). La equidad de género es un pilar fundamental de la interdisciplina, por lo que solo con una disminución de la brecha de género se podrá avanzar adecuadamente en este sentido.

Creemos, junto con Vianello y Caramazza (2002), que el aumento de la presencia de una mirada crítica, que incluya esta perspectiva de género desde los diversos feminismos en las instituciones, en la academia, en las organizaciones y en la comunidad, generará otras formas (quizás novedosas), más democráticas, abiertas e inclusivas que las existentes. No obstante, ello supondrá el ejercicio arduo y no menos exigente, de desandar estructuras, formatos y formaciones socio-culturales hegemónicas, naturalizadas y legitimadas en nuestras sociedades. En este sentido, Vianello y Caramazza fundamentan la importancia de integrar visiones desde la equidad, la diversidad y la diferencia para aceptar lo que de válido pueda haber en la diferencia y luchar para eliminar la supremacía masculina, que es eminentemente destructiva, con la vista puesta en un nuevo orden de cosas donde "los elementos positivos que son y seguirán siendo diferentes en uno y otro género (...) puedan afirmarse en un contexto igualitario: es decir, de respeto mutuo y constructivo" (Vianello y Caramazza, 2002, p. 23).

Complementariamente, la UDELAR y PEDECIBA como principal programa de desarrollo de ciencias básicas en nuestro país, son espacios de generación de conciencia crítica y clave para la construcción de conocimiento transformador. La baja representatividad de las mujeres, disidencias sexuales, dominancia del patriarcado, la ausencia de perspectiva de género y escaso reconocimiento de aportes de estudios feministas en los programas de posgrado resultan, además, en la reproducción de los estereotipos en contenidos, prácticas y metodologías. Por esta razón, es fundamental aplicar medidas transversales y específicas a fin de incorporar la perspectiva de género en estos ámbitos para mejorar el sistema de investigación actual y prepararlo para las nuevas generaciones. De no revisarse los procesos actuales y no generar alternativas, se observarán pérdidas para las estudiantes e investigadoras, para el desarrollo de las Geociencias en general, así como también para las áreas STEM que se encuentran actualmente en plena expansión y son clave en los procesos de desarrollo de los países. El análisis de cómo la desigualdad originada en la Universidad se transfiere al ámbito laboral público o privado y luego se transforma en una desigualdad estructural, sería una perspectiva de este trabajo.

\section{Agradecimientos}

Agradecemos a las y los integrantes del área PEDECIBA-Geociencias, al Sistema Nacional de Investigadores de la Agencia Nacional de Investigación (SNI-ANII) y muy especialmente al equipo revisor y editor de la revista por su gran aporte a mejorar el manuscrito.

\section{ReFERENCIAS}

Bernheim, R. (2015). Informe de Género. Documentos de Discusión $N^{\circ} 1$. Montevideo: Unidad de Evaluación y Monitoreo. Agencia Nacional de Innovación e Investigación. Recuperado a partir de: https://www.anii.org.uy/ upcms/files/listado-documentos/documentos/informe-g-nero-2015.pdf

Bielli, A., Buti, A. y Viscardi, N. (2002). Mujeres investigadoras uruguayas: ¿participación real o incierta? Documento de Trabajo $N^{\circ}$ 9. Montevideo: Comisión SectoriaI de Investigación Científica. Universidad de la República de Uruguay. 
Bourdieu, P., Passeron, J. C., Melendres, J., y Subirats, M. (1977). La reproducción: elementos para una teoría del sistema de enseñanza. Barcelona: Laia.

Bukstein, D. y Gandelman, N. (2017). Glass ceiling in research: Evidence from a national program in Uruguay. Working Paper Series 798. Inter-American Development Bank.

Cárdenas Tapia, M. (2015). La participación de las mujeres investigadoras en México. Investigación administrativa, 44(116). Recuperado a partir de: http://www.scielo.org.mx/scielo.php?script=sci_arttext\&pid=S2448-76782 015000200004\&lng=es\&tlng=es

Cech, E. A. y Blair-Loy, M. (2010). Perceiving glass ceilings? Meritocratic versus structural explanations of gender inequality among women in science and technology. Social Problems, 57(3), 371-397. DOI: https://10.1525/s p.2010.57.3.371

Consejo Nacional de Género (2020). Estrategia Nacional para la Igualdad de Género. 2030. Ministerio de Desarrollo Social. Instituto Nacional de las Mujeres. Recuperado a partir de: https://www.gub.uy/ministerio-desarrollo-s ocial/node/1941

Cotter, D. A., Hermsen, J. M., Ovadia, S. y Vanneman, R. (2001). The glass ceiling effect. Social forces, 80(2), 655-681. Recuperado de www.jstor.org/stable/2675593

De Welde, K. y Laursen, S. (2011). The glass obstacle course: Informal and formal barriers for women Ph. D. students in STEM fields. International Journal of Gender, Science and Technology, 3(3), 571-595. Recuperado a partir de http://genderandset.open.ac.uk/index.php/genderandset/article/view/205

Eagly, A. H., y Carli, L. L. (2007). Through the labyrinth: The truth about how women become leaders. Harvard Business Press.

Etchebehere, C. (2019). 42\% de mujeres en el SNI: ¿un logro o una gran inequidad? La Diaria. Recuperado a partir de https://ladiaria.com.uy/articulo/2019/4/42-de-mujeres-en-el-sni-un-logro-o-una-gran-inequidad/

Etzkowitz, H., Kemelgor, C., Neuschatz, M., Uzzi, B. y Alonzo, J. (1994). The Paradox of Critical Mass for Women in Science. Science, New Series, 266(5182), 51-54.

Ferrari, N. C., Martell, R., Okido, D. H., Romanzini, G., Magnan, V., Barbosa, M. C. y Brito, C. (2018). Geographic and gender diversity in the Brazilian Academy of Sciences. Anais da Academia Brasileira de Ciências, 90(2). htt p://dx.doi.org/10.1590/0001-3765201820170107

Fraser, N. (1997). Iustitia interrupta: reflexiones criticas desde la posición "postsocialista". Bogotá: Siglo del hombre editores.

Frins, E., Auyuanet, A., Cabeza, C., Stari, C., Kahan, S. y Renom, M. (2015). The presence of women in physics in Uruguay. AIP Conference Proceedings. https://doi.org/10.1063/1.4937693

Guevara Ruiseñor, E., Medel Figueroa, D. y García, C. C. (2012). Las académicas como modelo para dedicarse a la investigación en estudiantes de psicología. Revista Mexicana de Orientación Educativa, 9(23), 36-42. Recuperado a partir de: http://pepsic.bvsalud.org/scielo.php?script=sci_arttext\&pid=S1665-75272012000200006\&lng= pt\&tlng=es

Hernando, A. (2015). Mujeres, hombres, poder. Subjetividades en conflicto. Madrid: Traficantes de Sueños.

Holmes, M. A., O'Connell, S. y Dut, K. (Eds.). (2015), Women in the Geosciences: Practical, Positive Practices Toward Parity. New Jersey: American Geophysical Union. John Wiley \& Sons. Book Series.

Martinis, P. (2012). Prólogo. En La violencia está en los otros. La palabra de los actores educativos (pp. 7-9). Montevideo: Trilce.

Morrison, A. M., White, R. P., y Van Velsor, E. (1987). Breaking The Glass Ceiling: Can Women Reach The Top Of America's Largest corporations? New Jersey: Pearson Education.

Nielsen, M. W., Alegria, S., Börjeson, L., Etzkowitz, H., Falk-Krzesinski, H. J., Joshi, A., Leahey, E., Smith-Doerr, L., Williams Woolley, A. y Schiebinger, L. (2017). Opinion: Gender diversity leads to better science. Proceedings of the National Academy of Sciences, 114(8), 1740-1742. DOI: https://10.1073/pnas.1700616114 
Oficina de Planeamiento y Presupuesto (2017). Mujeres en ciencia, tecnología, e innovación, un problema de justicia. Montevideo: Presidencia República Oriental del Uruguay. Recuperado a partir de: https://www.miem.gub.uy/ sites/default/files/mujeres_en_ciencia_tecnologia_e_innovacion.pdf

Osborn, M. (1994). Status and Prospect of Women in Science in Europe. Science New Series, 263(5152), 1389-1391.

Osborn, M. (2008). Cómo lograr la equidad de género en ciencia. Revista de la Sociedad Española de Bioquímica y Biologia Molecular, 158, 10-14.

PEDECIBA-Geociencias (2018). Aportes científicos y de gestión académica al Área de Geociencias. Informe final Jornadas Geociencias IX. Mimeo.

Rovetto, F. y Figueroa, N. (2017). "Que la universidad se pinte de feminismos" para enfrentar las violencias sexistas". Descentrada, 1(2), e026. Recuperado de http://www.descentrada.fahce.unlp.edu.ar/article/view/DESe026

Serio, T. (2016). Speak up about subtle sexism in science. Nature, 532(7600), 415-415.

Shen, H. (2013). Inequality quantified: Mind the gender gap. Nature News, 495(7439), 22-24.

Tang, J. (1997). The glass ceiling in science and engineering. The Journal of Socio-Economics, 26(4),383-406. https:/ /doi.org/10.1016/S1053-5357(97)90003-2

Tomassini Urti, C. (2012). Ciencia académica y género: trayectorias académicas de varones y mujeres en dos disciplinas del conocimiento dentro de la Universidad de la República. Montevideo: Ediciones Universitarias.

Unidad de Mujeres y Ciencia (2018). Cientificas en cifras 2017. Estadisticas e indicadores de la (des)igualdad de género en la formación y profesión científica. Madrid: Ministerio de Ciencia, Innovación y Universidades. Recuperado a partir de: https://www.ciencia.gob.es/stfls/MICINN/Ministerio/FICHEROS/UMYC/Cientificas_cifras_20 17.pdf

Van Vlooten, D. (2005). Cutting the Gender Scissors. Science. Recuperado a partir de: https://www.sciencemag.org /careers/2005/02/cutting-gender-scissors

Vianello, M. y Caramazza, E. (2002). Género, espacio y poder. Para una crítica de las Ciencias Politicas. Madrid: Cátedra - Universitat de València.

Vila-Concejo, A., Gallop, S. L., Hamylton, S. M., Esteves, L. S., Bryan, K. R., Delgado-Fernandez, I., y Power, H. E. (2018). Steps to improve gender diversity in coastal geoscience and engineering. Palgrave Communications, 4(103). https://doi.org/10.1057/s41599-018-0154-0

Viscardi, Nilia (1998) Violencia en el espacio escolar. Revista Relaciones, 172, 5-7, Montevideo.

Viscardi, Nilia (1999) Violencia en el espacio escolar. Prácticas y representaciones (Tesis de Maestría). IFCH-UFRGS, Porto Alegre.

Viscardi, Nilia (2003) Enfrentando la violencia en las escuelas: un informe de Uruguay. En: Violencia na Escola: América Latina e Caribe (pp. 153-205). Brasilia: Unesco.

Viscardi, Nilia (2008) Violencia en las aulas: práctica educativa, conflicto escolar y exclusión social. En Paternain, Rafael y Sanseviero, Rafael (comps.) Violencia, inseguridad y miedos en Uruguay. ¿Qué tienen para decir las Ciencias Sociales? (pp. 145-160) Montevideo: FESUR.

Williams, J. C. y Segal, N. (2003). Beyond the Maternal Wall: Relief for Family Caregivers Who Are Discriminated Against on the Job. Harvard Women's Law Journal, 26, 77-162.

Williams, J. C. (2005). The glass ceiling and the maternal wall in academia. New Directions for Higher Education, 130. https://doi.org/10.1002/he.181

Wright, K. B. (2005). Researching Internet-based populations: Advantages and disadvantages of online survey research, online questionnaire authoring software packages, and web survey services. Journal of computermediated communication, 10(3). https://doi.org/10.1111/j.1083-6101.2005.tb00259.x

Yáñez, S. (2016). Trayectorias laborales de mujeres en ciencia en tecnologia: barreras y desafios. Un estudio exploratorio. Chile: FLACSO. 


\section{Apéndice I}

Listado de preguntas binarias y múltiple opción de la encuesta realizada a los y las integrantes del área de Geociencias de PEDECIBA.

1. ¿Crees que en Uruguay se discrimina negativamente a las mujeres en el mercado laboral? Sí / No

2. ¿Crees que en la Universidad de la República se discrimina negativamente a las mujeres? Sí / No

3. ¿En qué sentido crees que las mujeres son discriminadas en el trabajo?

Menor salario / Peores puestos de trabajo / Privación de la promoción en el trabajo / Falta de respeto por parte de los compañeros / Cuestiones legales / Es más difícil encontrar trabajo/No están preparadas para trabajos cualificados

4. ¿Crees que en el área PEDECIBA Geociencias existen diferencias entre mujeres y hombres? Sí / No

5. ¿Crees que en el área de Geociencias existe un techo de Cristal? Sí / No

6. ¿Cuál crees qué es la primera medida a tomar para mejorar la situación de la mujer dentro del área? Sí / No

Que exista equidad en las comisiones / Que se considere la maternidad /Que los proyectos incluyan un cupo mínimo de mujeres participantes

7. ¿Estás de acuerdo en tener consideraciones respecto a la maternidad?

8. ¿Crees que es necesario tomar acciones concretas en cuestiones de género dentro del área? Sí / No

\section{Notas}

1 Para mayor información, remitimos a http://pedeciba.edu.uy/geocien/

2 Ver: pedeciba.edu.uy/investig-geo

\section{BY-NC-SA}

